\title{
A Review on Home Automation using Augmented Reality
}

\author{
Pranav Bedekar ${ }^{1}$, Snehal Nargundi ${ }^{2}$ \\ ${ }^{1}$ Student, IT Department, RMD Sinhgad School of Engineering \\ ${ }^{2}$ Guide, IT Department, RMD Sinhgad School of Engineering
}

\begin{abstract}
This paper presents the use of augmented reality in the field of home automation. Augmented reality increases the interactive control of the devices and appliances with the users operating them. Now a days it is possible to communicate and control the appliances remotely with the emergence of smart devices. Many methods are used to control the appliances remotely but it becomes difficult for the users to use the smart devices because of the GUI(Graphical User Interface) control. For this solution, we use augmented reality (AR) technology for easier operation of home appliances connected by home networks. AR technology can provide virtual graphics and apply additional information to specific areas through camera displays. This paper presents how augmented real ity is used for controlling home appliances.
\end{abstract}

Keywords: Augmented reality, home automation, voice control, raspberry pie

\section{Introduction}

With the advent of technology, there is a wide increase in the use of smart phones, laptops and people are getting more and more dependent on these devices for doing their work. People can control and handle different appliances through their devices using different techniques. Augmented reality(AR) is one of the recent technology evolved for automation of the electrical appliances. This technology gives a virtual view of the devices generating a real environment (see figure 1).

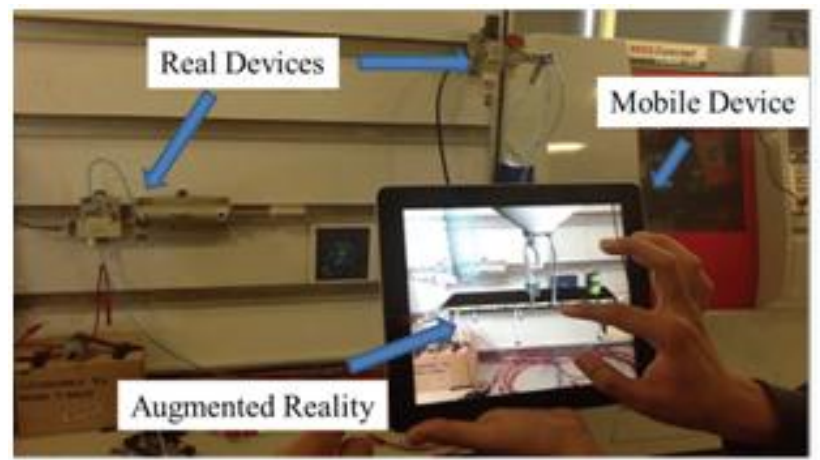

Figure 1: Example of augmented reality

Augmented Reality brings virtual objects into the real world where we live. Augmented reality is used in many areas such as navigation in real-world environments, advertising, military, emergency services, art, games, architecture, sightseeing, education, entertainment, commerce, information visualization, translation and so on. What has really brought Augmented Reality to life in the recent years is that AR applications are now available on many smart devices and are easy to use.In principle, AR can be implemented on any computer and handheld device that use video-see through technology that allows to "see through" the display to view both the real world and superimposed computer-generated objects.

With advancement of Automation technology, life is getting simpler and easier in all aspects. In today's world Automatic systems are being preferred over manual system. Internet has played a major role in the field of automation. Internet of things is a growing network for controlling the home appliances such as lights, fans ,Television, etc. Home Automation system using IoT is a system that uses computers or mobile devices to control basic home functions and features automatically through internet from anywhere around the world. An automated home is sometimes called a smart home. Many a times people forget to switch off their electrical appliances when they leave their home. But due to the use of smart phones to control the home appliances this problem has been reduced.

Augmented reality usage in the field of home automation is a relatively new idea and there has been a growing interest regarding its implementation. The usage of AR gives us significant advantage over other models since it can help us to control devices or machines in a real time environment and helps anyone understand how to operate them. Using Augmented reality ,the users are able to control the appliances more effectively and easily. Augmented reality gives the users a virtual experience which help them identify the different switches through their mobile devices.

\section{Proposed System}

\section{A. Introduction}

The proposed system aims to assist people, especially the disabled and elderly who cannot do their own work and need help from others for doing simple tasks such as controlling the electrical appliances in the home. They are dependent on others which causes a lot of inconvenience to many people. With the emergence of smart home devices and easy use of mobile devices people can communicate and control the home appliances. But in many cases the GUI(Graphical User Interface)of the mobile is difficult to use for the disabled people. This system uses Augmented Reality (AR) technique for automation which is getting increasingly popular for controlling of home appliances. 
Using this technology it will greatly help the disabled and elderly people to control the appliances easily. A mobile application along with ARCH Server will help the users to control a switch by simply pointing their mobile camera to it from a distance. Different virtual switches will appear when the camera is pointed to different appliances, thus allowing the user to control different appliances easily and conveniently. Instead of 2D buttons,3D switches will appear on the screen which gives a familiar interface to the user. Voice Control technology will also be used so that the users can control the appliances using voice commands. A remote web browser interface will also be implemented which will allow the users to control the appliances through Internet even if they are not present at home..

\section{B. System Architecture}

In the proposed system Augmented Reality (AR) is used to allow a virtual object to pop out on the mobile screen when the user points his camera towards the object or a switch. For using AR, Wikitude SDK is required. Wikitude SDK is a software library and framework for mobile apps used to create augmented reality experiences. Using an augmented reality application, a virtual image is mounted over the live camera image of the real world. Augmented reality enhances the experience of the user. When the user points the camera towards a switch, a 3D image will pop up on the screen and the user can turn on or off the switch using the AR buttons present on the screen. AR uses the concept of image tracking, processing and communicating with backend server (ARCHServ) to control the applications. Arch Server is a low cost and efficient linux based server .It is used for handling all the appliances at the backend.

Voice control technique is also implemented which uses android speech recognition so that the users can control the appliances through the voice commands.For voice control user only has to select the option of the appliance and control status through voice commands. When a user speaks a command the voice is captured through the microphone. Once the voice is captured, the input voice is compared with the data stored earlier before testing. The output of the comparison is the voice matched with any of the command trained and certain signal is produced as the input for controlling system.

A remote web control feature is used to enhance the operation of the appliances over the mobile devices. Control data is sent to ARCHServ to activate the General Purpose Input Output (GPIO) pins which will then trigger the household DC or AC appliances (E.g. On/Off). The status of the appliances can be checked through web browser or through mobile web interface and control them using internet. An automated voice command will help the users to navigate to different options such as (option 1 for augmented reality, option 2 for voice control and option 3 for Remote web control.). The user only has to select the option through its voice for that activity to start.

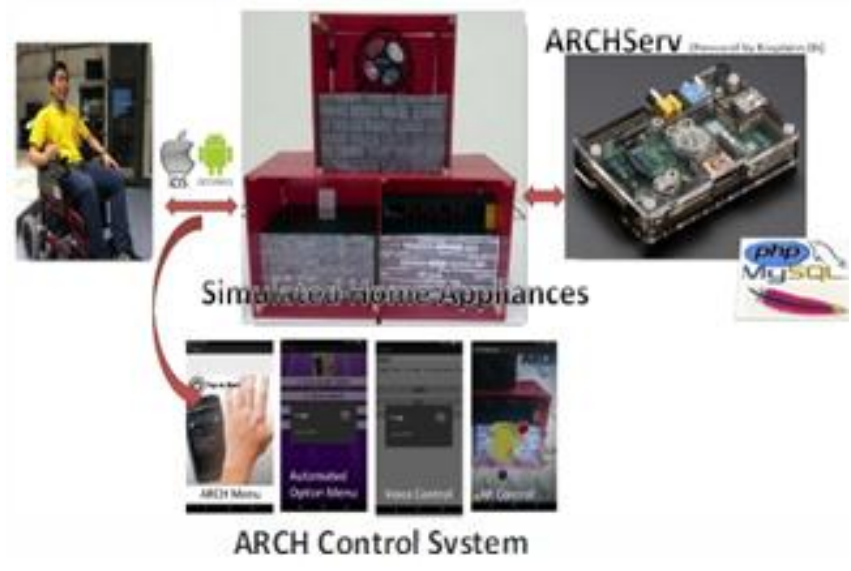

Figure 2: Proposed System

\section{Desired Outcomes}

As this system is mainly proposed for the disabled and elderly people, it is easy for them to control the appliances and be less dependent on the caretakers. This system can also be implemented at hospitals so it helps reduce the burden on the nurses and to decrease the hospital expenses. If the users forget to switch off the appliances, they can do so remotely with their mobile devices and hence save electrical power and reduce electrical hazards. As Augmented reality is used ,it will help the users with easy navigation and interactive control of electrical appliances. Thus this system is believed to control and monitor the electrical usage and switch off appliances with ease.

\section{Hardware and Software Specifications}

\section{A. Hardware Specifications}

Raspberry pie is used for the backend processing which provides an interface for the electrical appliances and the mobile devices. It is also used for hosting web, MySQL and GPIO servers. Raspberry pie is a small size fully functional computer capable of running a Linux based operating system. To interface raspberry-pi with the external world we can use WebIOPi. WebIOPi is a web application which allows to control Raspberry Pi's GPIO. Different electrical appliances such as fan, light bulb, Television, etc are used which are controlled by users using the mobile devices.

\section{B. Software Specifications}

A mobile application (android/ios) is used for controlling the electrical appliances. This application will use the camera of the mobile to point to the different virtual switches of the appliances. A mobile web control interface is used to control the appliances using internet when users are not present at home.

ARCH server is used as a backend server for communicating with the appliances. Arch is a low cost linux based server which can control the GPIO pins that will trigger the household appliances.

\section{Conclusion}

This paper describes the use of Augmented reality to control the home appliances. With the use of smart phone devices 
users will be able to switch on/off the electrical appliances through internet or home network .This system also proposes features such as voice control and remote web control interface for controlling the appliances. This system will mainly help the disabled and elderly people to control the appliances giving them a real world experience with the help of Augmented reality.

\section{Acknowledgments}

I would like to take this opportunity to express my profound gratitude and deep regard to my guide, Mrs. Snehal Nargundi, Information Technology Department, RMDSSOE and the Academic Staff of the Department for their exemplary guidance, valuable feedback and constant encouragement and support.

\section{References}

[1] Evil Genius, McGraw-Hill/TAB Electronics. "Raspberry Pi Projects for the Evil Genius", 1'1 Edition, 2014.

[2] 2014, Autodesk, Inc, Web Control of Raspberry Pi GPIO, accessed on 22 Jan 2015. http://www.instructables.comlid/WebControl-ofRaspberry-Pi-GPIOI

[3] Kimmo Karvinen \& Tero Karvinen, Make: Getting Started with Sensors, Measure the world with Electronics, Arduino and Raspberry Pi, 1 st Edition, 2014

[4] Michal Varchola, Milos Drutarovsky, "Zigbee based home automation wireless sensor network" IEEE PERCOM Workshops, pp. 141-146, 2011.

[5] Leroy Zi Wei Tang, Kian Sin Ang, Mohamad Amirul Maricar Bin Mohamed Yusoff, "Augmented Reality Control Home (ARCH) for Disabled and Elderlies" 2015 IEEE Tenth International Conference on Intelligent Sensors, Sensor Networks and Information Processing (ISSNIP)

[6] K. Bromley, M. Perry, and G. Webb. "Trends in Smart Home Systems,Connectivity and Services", www.nextwave.org.uk, 2003.

[7] 2015 Wikitude GmbH, Wikitude AR project, accessed 22 Jan 2015. http://www.wikitude.com/app 